\title{
Towed Ship Motion Test in Directional Spectrum Waves in a Long Tank (Part 3)
}

\author{
by Seiji Takezawa*, Member Tsugukiyo Hirayama*, Member \\ Shivashis Acharyya**, Member
}

\begin{abstract}
Summary
As a sequel to our previous reports on tank testing results of ship motions in directional spectrum waves this paper discusses the applicability of motion test results to estimate transfer functions of motions for various wave encounter angles. As we have observed in our previous reports that in many cases, especially in head sea condition, theoretical estimation of motion spectra do not agree so well with experimental results, estimation of true magnitude of transfer functions becomes a necessity. In the present study a practical method of estimation has been discussed and short term prediction of motions over various range of wave periods has been carried out with the estimated transfer functions. The prediction shows good agreement with experimental results. Results of long term prediction in Winter North Atlantic have also been presented.

This paper also discusses the possibility to extend Maximum Likelihood Method (MLM) to estimate directional encounter wave spectra analyses of which is necessary for reverse calculation of transfer functions of motions.
\end{abstract}

\section{Introduction}

Strip Method has long been in use to estimate ship motions in various wave conditions. However validity of this method over various ship forms and over different speed range is still a topic of debate among the scientists and engineers. The actual sea state is of short crested in nature. Because of limitations to generate short crested waves (here we shall call this as directional spectrum waves) in a towing tank, ship motions under this condition could not be investigated for a long time. For the first time we have carried out running tests in directional spectrum waves in the towing tank of Yokohama National University. These test results have been presented in our previous reports ${ }^{1), 2}$. We have observed that theoretically estimated ship motion spectra have non-negligible difference compared of experimental results, especially under head sea condition. Results from long crested transient water wave experiments clearly show that Strip Method over-estimates transfer functions of heave and pitch and underestimates that of roll, especially in the region of natural frequency of each mode of motion. If the transfer functions are wrongly estimated further calculations to predict ship motions over various range of mean wave

\footnotetext{
* Faculty of Engineering, Yokohama National University.

** Graduate School, Yokohama National University.
}

periods and wave directions become meaningless, even if we consider that the linearity assumption is valid.

In a conventional long towing tank we have limitations over mean wave direction with respect to the model, during running tests. The transfer functions of motions (apart from roll) in head and following sea can be estimated directly from long crested transient water wave experiments; but variation of transfer functions of motions with wave encounter angle remains unknown except for a few experimental results obtained in rectangular model basin. As already mentioned, the true magnitude of transfer functions at different wave encounter angle need to be determined to calculate responses in directional spectrum waves. In the present paper we shall discuss a practical method of estimating directional transfer functions of motions from motion test results with New Strip Method as the base and also discuss the applicability of this results to short term and long term predictions. It should be noted that in our study, surge, sway and yaw motions are fixed.

\section{Estimation of Transfer Functions of Motions}

\section{1 Calculation Method}

The basic equation to relate motion spectrum and wave spectrum is given by:

$$
S_{z}\left(\omega_{e}, \chi\right)=S_{5}\left(\omega_{e}, \chi\right)\left|H_{z}\left(\omega_{e}, \chi\right)\right|^{2}
$$

where, subscript $z$ means motion, for example heave, $\zeta$ means wave. $S=$ Spectrum, $H=$ Transfer Function.

$\omega_{e}=$ Encounter wave frequency, $\chi=$ Wave encounter angle. 
Representation of equation ( 1 ) is in the form of directional spectrum. To obtain one-dimensional frequency spectrum we need to integrate this equation over wave encounter angle as follows:

$$
S_{z}\left(\omega_{e}\right)=\int_{-\pi}^{\pi} S_{\zeta}\left(\omega_{e}, \chi\right)\left|H_{z}\left(\omega_{e}, \chi\right)\right|^{2} d \chi
$$

For each frequency level the above equation can be discretized as :

$$
S_{z}=\sum_{i=1}^{N_{\chi}} S_{\zeta_{i}} H_{z i}^{2} \Delta \chi
$$

where $N_{x}=$ No. of wave encounter angle.

We can construct similar equations for different set of wave data. For example, for $M$ set of wave data we can obtain $M$ set of motion spectra and these $M$ equations can be expressed in matrix form as follows:

$$
\left\{S_{z}\right\}=\left[S_{\zeta}\right]\left\{H_{z}^{2}\right\} \Delta \chi
$$

where [ ] means $M \times N_{\chi}$ matrix and \{ $\}$ means $N_{x} \times 1$ column vector.

During a towing tank experiment ship motions are measured in the form of time histories from which we can obtain one-dimensional frequency spectrum by any standard method of spectral analysis like FFT or MEM. Also from the measured time histories of wave elevation during the experiment, the directional wave spectra can be estimated quite accurately, thanks to recent developments on this field ${ }^{3), 4}$. Thus in equation (4) we have $H_{z}$ as the only unknown parameter. Apparently, the equation (4) can be solved for $H_{z}^{2}$ if $M=N_{x}$ i. e. if the number of observations or experiments (which is equal to number of wave dataset) equals number of wave encounter angle. The number of wave encounter angle can be arbitrarily adjusted during MLM analysis of directional wave spectra; however directional resolution imposes a limitation on this number to be kept at some minimum value. But to arrive at the ideal situation of $M=N_{x}$ and to solve equation (4) by direct matrix inversion we have following limitations:

a) During the running test, if the towing tank is short the length of measured time history is not long enough and the wave data may not have sufficient frequency elements within this short period. Thus it is not possible to obtain a stable spectrum from the data measured in a single run. We need to carry out a number of running tests over separate signal lengths of same characteristic wave. The final spectrum can be obtained by taking average of spectra analyzed in separate observations ${ }^{1,2)}$. Thus a single equation similar to that of (2) is basically a result of a few running tests. To obtain fairly reasonable directional resolution, directional spectrum of wave need to be analyzed at least at 10 degrees intervals; the number of wave encounter angle thus counts 10, in this case (from 90 to 180 deg., considering the symmetrical character of transfer functions). Therefore to satisfy the above condition of $M=N_{\chi}$ the number of observations will count in the range of 50 to 100 depending on speed conditions. b) Before entering into this lengthy schedule of experiments we have examined equation (4) more thoroughly by computer simulation with standard wave spectrum and assumed transfer function data. It has been found that to increase the degree of accuracy of the result wave data-set should have distinctly different and fairly wide directional distribution with variation in mean wave directions. Standard directional distribution functions are in the form of cosine functions of different order. In the experiment also, the directional distributions of the waves are simulated to the order of cosine functions. They have similarity in form and the variations are not distinct enough. During the experiment in a random set of data it is quite impossible to obtain wave spectrum with absolutely different directional distribution. At any frequency level if any two dataset of wave has same directional distribution, the wave matrix $\left(S_{\zeta}\right)$ becomes singular and the equation (4) cannot be solved.

To solve equation (4) various kind of iterations can be thought of. New Strip Method provides fairly good estimation of nature of distribution of transfer function over wave frequency, if compared with test results in transient water wave. However the order of estimation by NSM does not agree quite well with experiment, especially at natural frequency of motion. Thus the true order of transfer functions can be approximated by multiplying a correction factor to the transfer function estimated by NSM. In the present study considering practicality, we shall assume that this correction factor is independent of wave encounter angle at a particular frequency level. Thus the solution of the equation (4) is approximated as :

$$
\left\{H_{z}^{2}\right\}=\left\{H_{z 0}^{2}\right\} \cdot \alpha
$$

where $H_{z 0}$ is the transfer function calculated by NSM. and $\alpha$ is a scalar.

Hence the error in above assumption is given by:

$$
\{\varepsilon\}=\left\{S_{z}\right\}-\left[S_{\zeta}\right]\left\{H_{z 0}^{2}\right\} \cdot \alpha \Delta \chi
$$

Therefore,

$$
\begin{aligned}
\varepsilon^{2}= & \{\varepsilon\}^{T}\{\varepsilon\} \\
= & \left\{S_{z}\right\}^{T}\left\{S_{z}\right\}-2 \alpha\left\{H_{z 0}^{2}\right\}^{T}\left[S_{\zeta}\right]^{T}\left\{S_{z}\right\} \cdot \Delta \chi \\
& +\alpha^{2}\left\{H_{z 0}^{2}\right\}^{T}\left[S_{\zeta}\right]^{T}\left\{S_{\zeta}\right]\left\{H_{z 0}^{2}\right\}(\Delta \chi)^{2}
\end{aligned}
$$

Applying condition of least square,

$$
\begin{aligned}
\frac{d \varepsilon^{2}}{d \alpha}= & -2\left\{H_{z 0}^{2}\right\}^{T}\left[S_{\zeta}\right]^{T}\left\{S_{z}\right\} \cdot \Delta \chi \\
& +2 \alpha\left\{H_{z 0}^{2}\right\}^{T}\left[S_{\zeta}\right]^{T}\left[S_{\zeta}\right]\left\{H_{z 0}^{2}\right\}(\Delta \chi)^{2}=0
\end{aligned}
$$

Thus,

$$
\alpha=\frac{\left\{H_{z 0}^{2}\right\}^{T}\left[S_{\xi}\right]^{T}\left\{S_{z}\right\}}{\left\{H_{z 0}^{2}\right\}^{T}\left[S_{\zeta}\right]^{T}\left[S_{\zeta}\right]\left\{H_{z 0}^{2}\right\}(\Delta \chi)}
$$

where superscript $T$ means transposition

Using this value of $\alpha$ we can estimate transfer functions of motions from equation (5). As this method of caluclation follows the reverse path of conventional method to estimate motion spectra, in the present paper we shall call this an estimation by back calculation or reverse calculation. 


\section{2 Directional Encounter Wave Spectra}

Analyzed motion spectra obtained from running tests are on encounter frequency base. To solve equation (4) with motion spectrum data we need to have directional wave spectra also on encounter frequency base. The technique of Maximum Likelihood Method (MLM) to analyze directjonal wave spectrum has been developed considering stationary position of wave gauge array ${ }^{5}$. So far in theoretical calculation, to obtain motion spectra applying equation ( 2 ), we have used encounter wave spectra transformed from absolute spectra analyzed from time history measured in rested condition $^{1), 2}$. As far as the total spectral energy and spectrum shape are concermed this method provides quite a reasonable estimation. However in order to apply equation (2) to obtain transfer function of motions one to one correspondence between spectral ordinates of motions and that of wave needs to be known rather than total spectral energy. In the present study we shall discuss applicability of MLM on wave data measured at running condition. Similar situation appears in ref. ( 7 ).
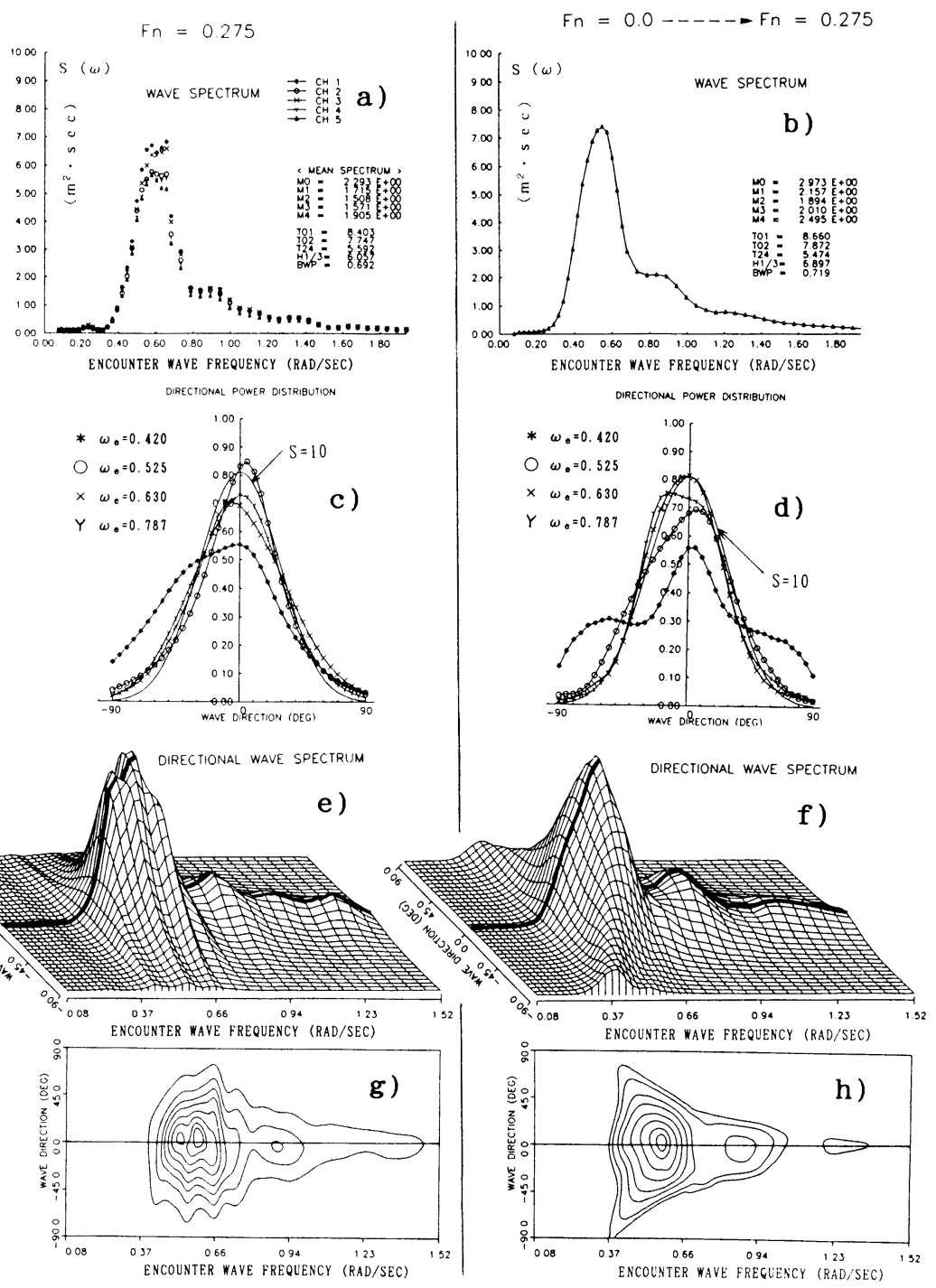

Fig. 1 Comparison between analyzed wave spectrum at $F n=0.275$ (left) and that transformed from spectrum at $F n=0.0$ (right). One dimensional spectrum ( $\mathrm{a} \& \mathrm{~b}$ ), Directional distribution at a few frequency level (c \& d), Two dimensional spectrum (e \& f) and corresponding contour curves ( $\mathrm{g} \& \mathrm{~h}$ ). 
The basic equation for Maximum Likelihood spectral estimation is given $b^{5)}$ :

$$
\begin{aligned}
S_{\zeta}\left(\omega_{0}, \chi\right)= & {\left[\sum _ { i = 1 } ^ { m } \sum _ { j = 1 } ^ { m } \phi _ { i j } ^ { - 1 } ( \omega _ { 0 } ) \operatorname { e x p } \left\{-i\left(k Y_{i j} \cos \chi\right.\right.\right.} \\
& \left.\left.\left.+k X_{i j} \sin \chi\right)\right\}\right]^{-1}
\end{aligned}
$$

where $\phi_{i j}^{-1}\left(\omega_{0}\right)$ denotes the $(i, j)$ components of the inverse of cross spectral matrix $\phi_{i j}\left(\omega_{0}\right)$.

$Y_{i j}, X_{i j}=$ Relative co-ordinates of the wave probes.

$k=$ Wave number.

The column vector consisting of the exponential term in this equation represents the phase difference among the wave components encountered by the wave sensors. This term needs special attention while analyzing wave data measured under running condition. Normally, wave number $k$ is estimated from the wave frequency by the following relation:

$$
k=\omega_{0}^{2} / g
$$

In running condition, due to the change in relative velocity of wave propagation with respect to wave sensors, the measured wave frequency (encounter frequency) differs from actual wave frequency (absolute frequency) and we need to convert the measured frequency to the order of absolute wave frequency in order to estimate wave number by relation $(9)$. The encounter frequency $\left(\omega_{e}\right)$ and the absolute frequency $\left(\omega_{0}\right)$ are related by following equation :

$$
\omega_{e}=\omega_{0}-\frac{\omega_{0}^{2}}{g} V \cos \chi
$$

where, encounter angle $\chi=180 \mathrm{deg}$. corresponds to head sea.

Applying this small correction equation ( 8 ) can be used without any change, to estimate directional encounter wave spectra except for following sea condi- tion.

Fig. 1 shows a comparison between a directional encounter wave spectrum from five wave probes at $F_{n}=$ 0.275 analyzed by the method mentioned above (left) and a encounter spectrum transformed from analyzed spectrum in rested condition (right) ; the wave characteristics being the same. We can find similarities in directional distributions at certain frequency levels

(Fig. 1 c \& 1 d) and in two dimensional representation of directional encounter spectra (Fig. 1 e \& $1 \mathrm{f}$ ) and their contour curves (Fig. $1 \mathrm{~g} \& 1 \mathrm{~h}$ ). The convex form of distribution of encounter wave energy over wave encounter angle may also be observed from both the set of contour curves. This clearly shows that the assumption we made to analyze directional encounter wave spectrum by MLM, as described above, is reasonable and we can obtain fairly good and practically usable results by this method. However we have limitations to apply this method to analyze directional encounter wave spectra in following sea due to the existence of overlapping of wave energy over certain frequency level $^{2)}$.

\subsection{Results}

The model under study, as mentioned in our previous reports ${ }^{1), 2)}$, is well known 'SR108' (or 'S7-175'), the model of a container ship and is the same as was used in ITTC comparative study. During the experiment with this model the surge, sway and yaw motions were fixed.

For this ship, results of transfer functions of heave, pitch, roll and vertical acceleration, estimated by the method we have introduced in section 2.1 , have been shown in Figures 2, 3, 4 and 5 respectively. In these
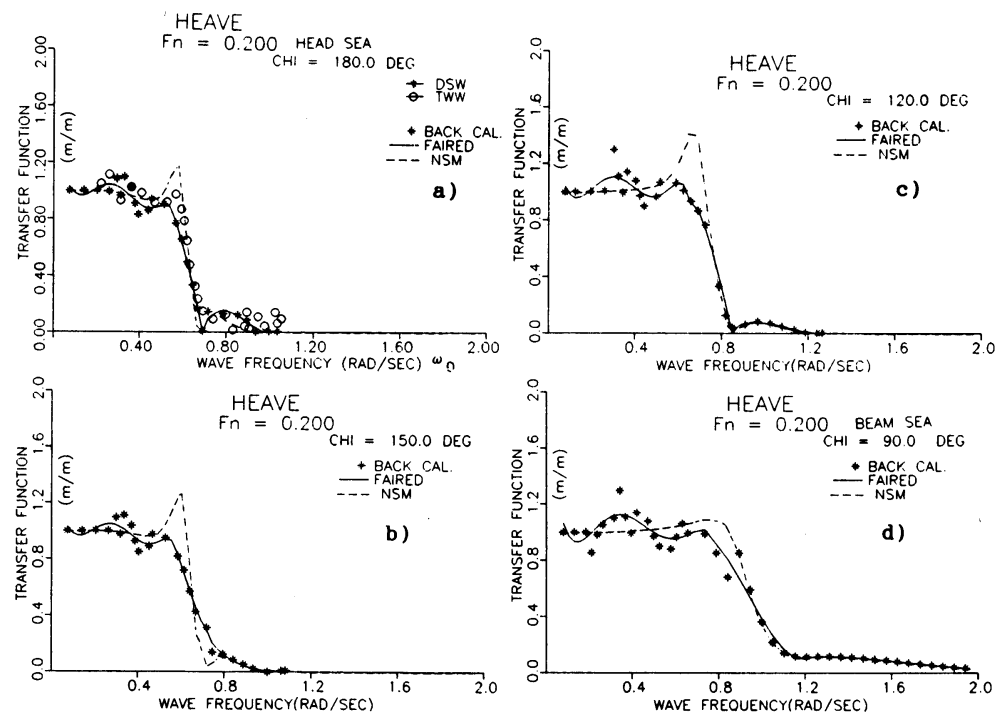

Fig. 2 Transfer Functions of Heave for wave encounter angles of $180.0 \mathrm{deg}$ ( a ), $150.0 \mathrm{deg}$. ( b ), $120.0 \mathrm{deg}$. (c) $90.0 \mathrm{deg}$. (d). 

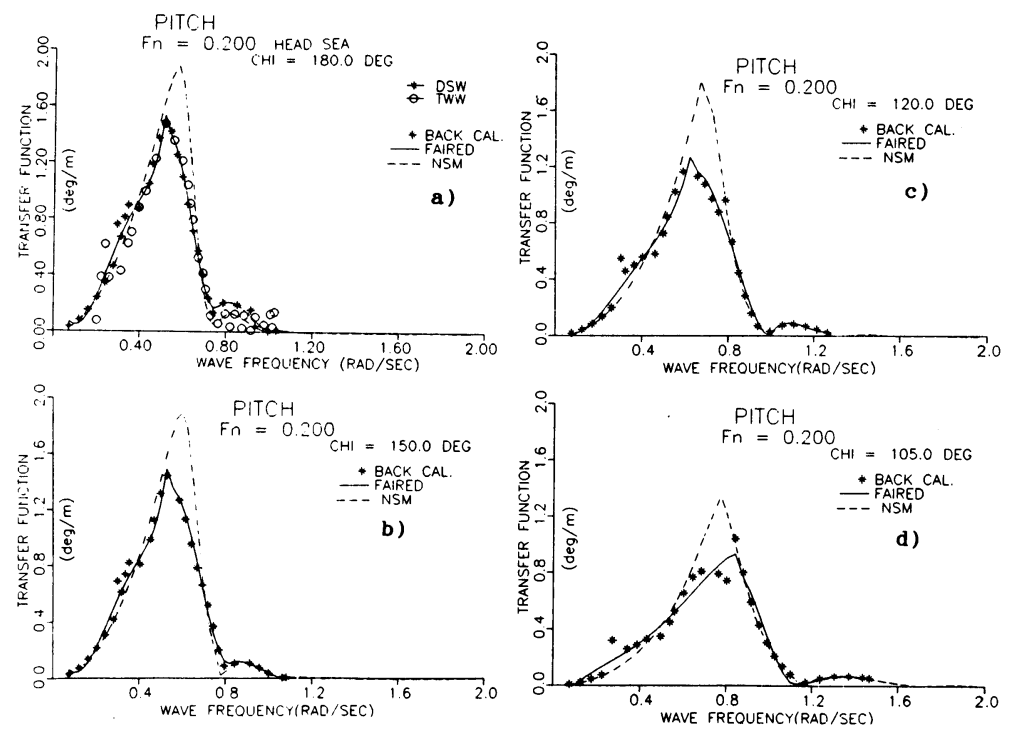

Fig. 3 Transfer Functions of Pitch for wave encounter angles of $180.0 \mathrm{deg}$ ( a ), $150.0 \mathrm{deg}$. ( b ), $120.0 \mathrm{deg}$. (c) $105.0 \mathrm{deg}$. (d).
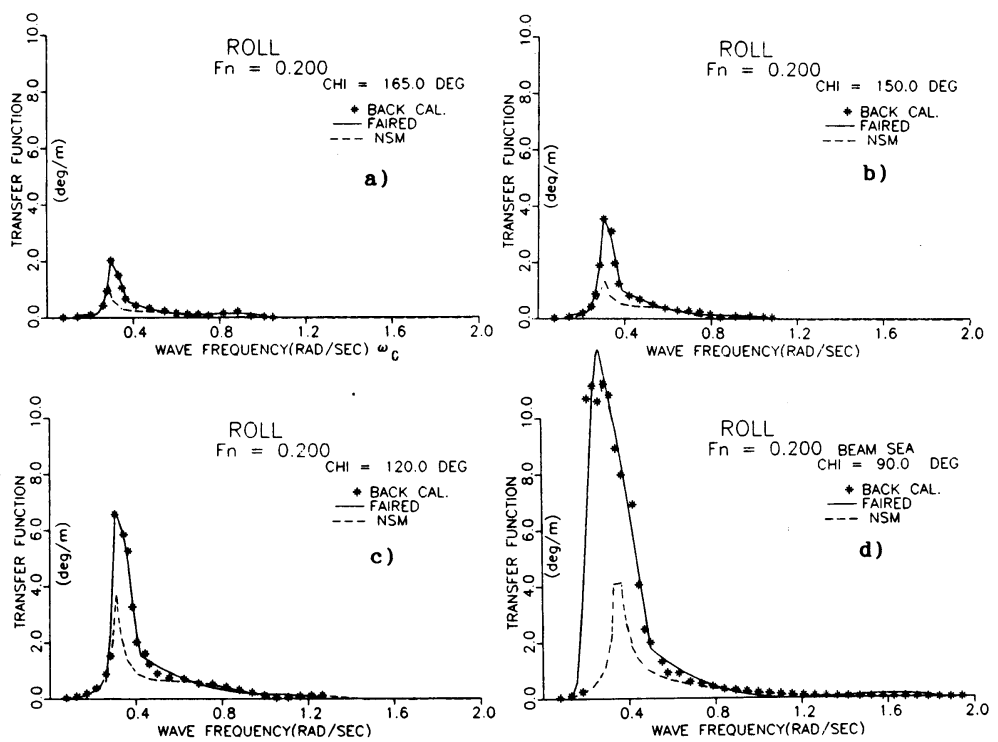

Fig. 4 Transfer Functions of Roll for wave encounter angles of $165.0 \mathrm{deg}$ ( a ), $150.0 \mathrm{deg}$. ( b ), $120.0 \mathrm{deg}$. (c ) $90.0 \mathrm{deg}$. (d).

figures ' $\mathrm{CHI}$ ' represents wave encounter angle and $\mathrm{CHI}=180.0 \mathrm{deg}$ has been taken as head sea. In the calculation we have imposed one condition such that if at any frequency level wave spectral density ordinate is less than $5 \%$ of the peak energy level the correction factor alpha $(\alpha)$ of section 2.1 will return a value of unity i. e. transfer function at that point will be assumed to be same as NSM estimation. This will have negli- gible effect on motion spectra as the wave energy is not of considerable order at this point. The points marked by '*' are the results of direct calculation. Due to scattering of these points at certain frequency levels we attempted to find a best fit curve by partwise regression analysis. This curve has been shown by the continuous line. The curve shown by broken line is the result of NSM. In head sea condition we have also the results of trans- 

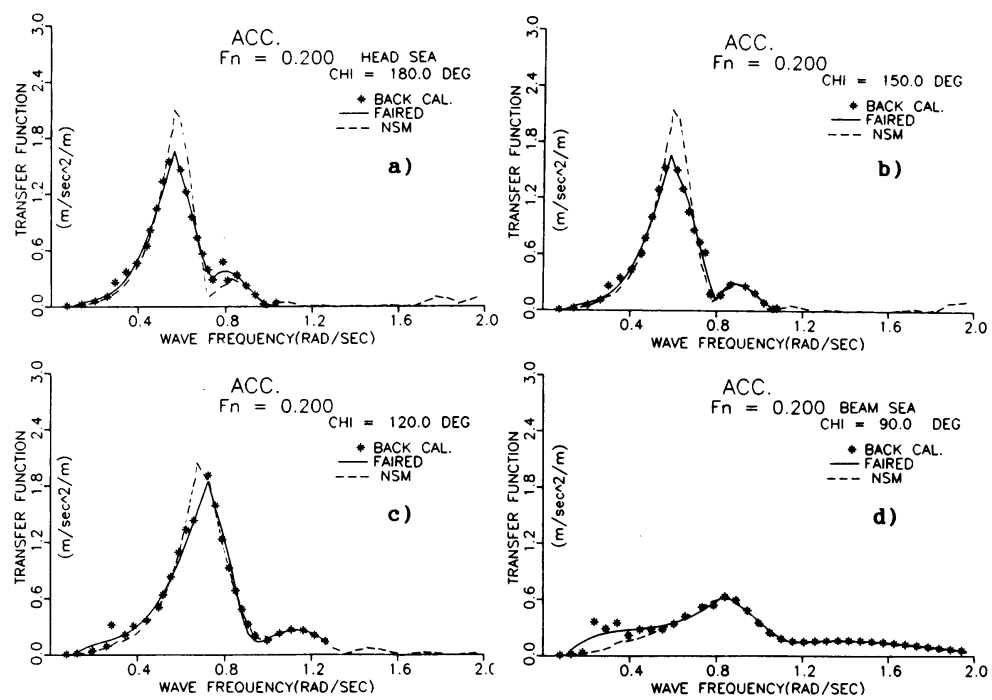

Fig. 5 Transfer Functions of Vert. Acc. for wave encounter angles of $180.0 \mathrm{deg}$ ( a ), $150.0 \mathrm{deg}$. ( b ), $120.0 \mathrm{deg}$. (c) $90.0 \mathrm{deg}$. (d).

fer functions from transient water wave (TWW) experiment. These results have been shown through the points marked by ' $O$ '. We can find a close similarity between the results of present approach and the experimental results of TWW suggesting the reliability of our method of estimation. The results of reverse calculation, if compared with NSM results, show fairly low value of transfer functions of heave, pitch and vertical acceleration at their natural frequency of motion (near the peak value). However in case of roll the estimated value of transfer functions are considerably high compared to calculated results by NSM. Although the results at Froude Number of 0.2 and at a few wave encounter angle have been presented here, similar results have been obtained for other wave encounter angles and at Froude Number of 0.275.

\section{Statistical predictions of ship motions}

In order to investigate on behaviour of a ship in all possible mean period of encounter in irregular sea waves, short term and long term predictions need to be carried out. In our previous report ${ }^{2)}$ we have presented the results of short term prediction in head and follow. ing sea using transfer functions of motions calculated by NSM. And we have observed, while comparing calculated results with experimental results, that this calculation led to over-estimation of heave, pitch and vertical acceleration in head sea and under-estimation of roll in both head and following sea condition. The results of heave, pitch and vertical acceleration in following sea however, were in good agreement with experimental results. Obviously the calculated results in head sea need corrections to the order of actual transfer function. Now with the new set of directional transfer functions, estimated from motion test results, we intend to carry out short term and long term predictions.

\section{1 Short Term Prediction}

A P-M type ITTC spectrum of the following form has been chosen for short term prediction.

$$
S_{5}\left(\omega_{0}\right)=\frac{A}{\omega^{5}} \exp \left(-B / \omega^{4}\right)
$$

The directional distribution function has been estimated by the two standard equations of following form :

$$
\begin{aligned}
& D_{1}(\chi)=C_{1} \cos ^{2 n} \chi \text { where, }-\frac{\pi}{2} \leqq \chi \leqq \frac{\pi}{2} \\
& D_{2}(\chi)=C_{2} \cos ^{2 s}\left(\frac{\chi}{2}\right) \text { where, }-\pi \leqq \chi \leqq \pi
\end{aligned}
$$

The directional wave spectrum has thus been approximated by :

$$
S\left(\omega_{0}, \chi\right)=S\left(\omega_{0}\right) D(\chi)
$$

The $s$ parameter of the equation ( $12 \mathrm{~b}$ ) represents the degree of directional spreading. In the present paper we have considered $s=10$ as a narrow spreading spectrum. For a wide spreading spectrum we have used equation ( 12 a ) with $n=1$, commonly known as 'cos square' distribution.

Two dimensional motion spectra have been estimated using above wave spectra and the transfer functions estimated in the present study by the following equation :

$S_{z}\left(\omega_{0}, \chi, V\right)=\left|H_{z}\left(\omega_{0}, \chi, V\right)\right|^{2} S_{\zeta}\left(\omega_{0}, \chi\right)$

where $S_{\xi}\left(\omega_{c}, \chi\right)=$ wave spectrum at $V=0$

It can be seen that in this expression, the mean direction of encounter wave is that of head sea, at the present case.

In Fig. 6 and Fig. 7 examples of calculated transfer 

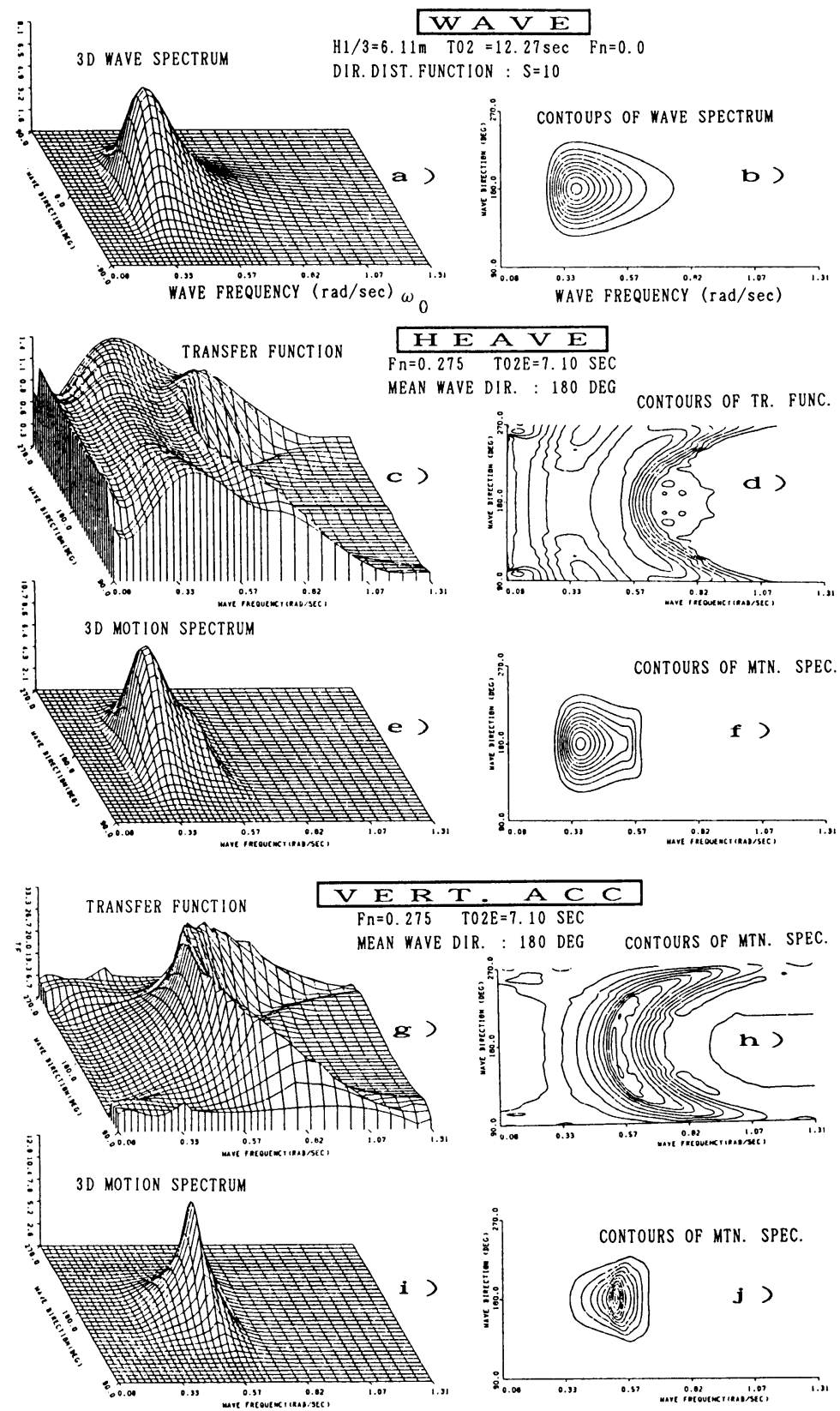

Fig. 6 Standard directional wave spectrum (a) at $F_{n}=0.0$ and corresponding contour curves $(b)$. Estimated transfer functions and corresponding contour curves of heave (c, d) and of vertical acceleration $(\mathrm{g}, \mathrm{h})$; Estimated motion spectra and corresponding contour curves of heave $(e, f)$ and of vertical acceleration $(\mathrm{i}, \mathrm{j})$; all at $F_{n}=0.275$. 

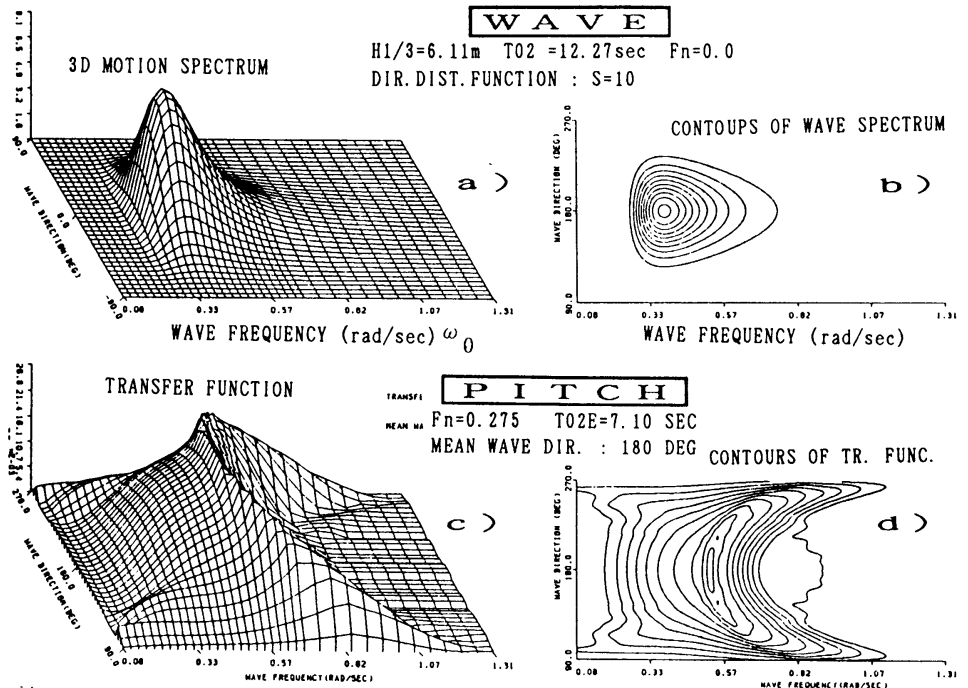

an $\mathrm{F} n=0.275$ T02E $=7.10 \mathrm{SEC}$ MEAN WAVE DIR. : 180 DEG CONTOURS OF TR. FUNC. c >
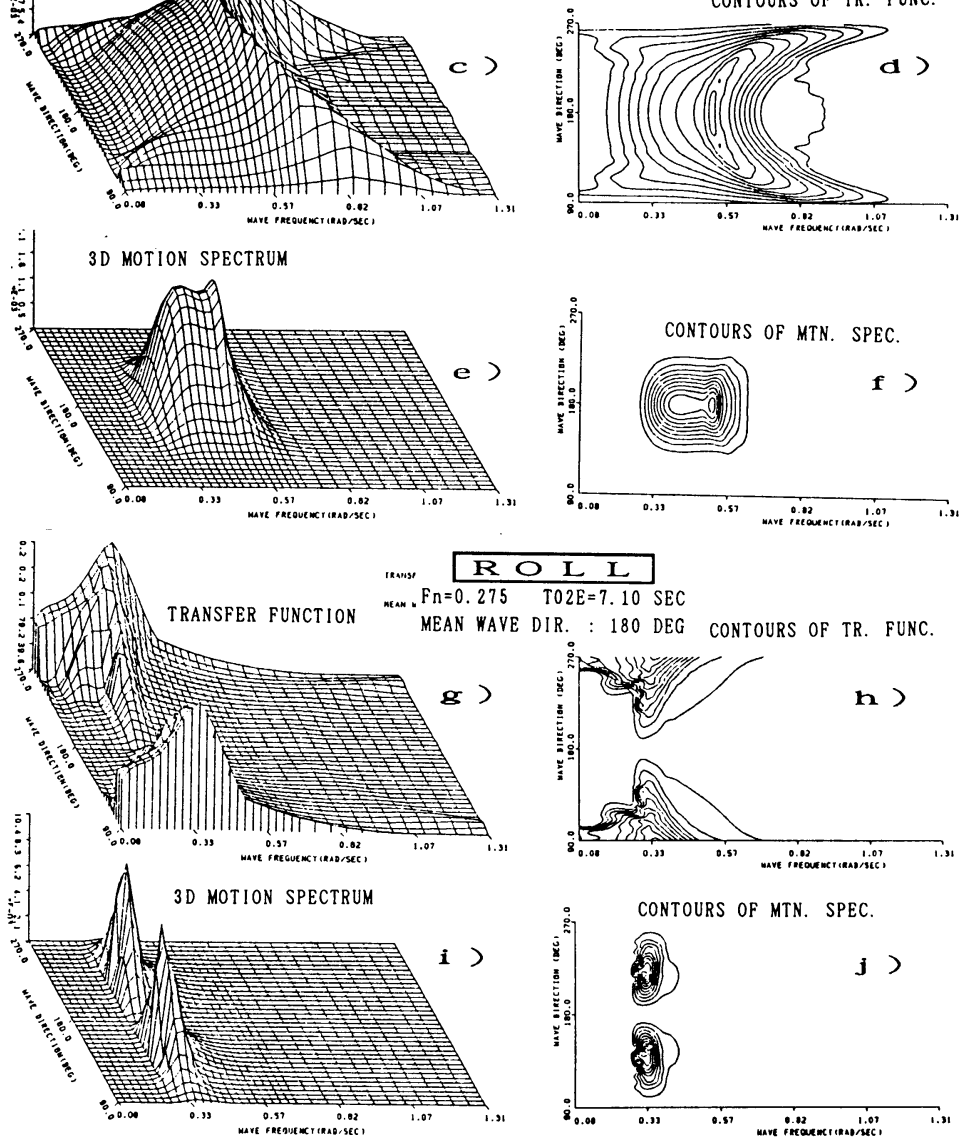

\section{T02E $=7.10$ SEC}

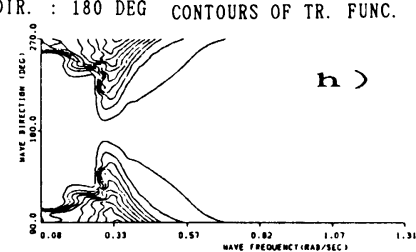

Fig. 7 Standard directional wave spectrum (a) at $F_{n}=0.0$ and corresponding contour curves ( $b$ ). Estimated transfer functions and corresponding contour curves of pitch (c, d) and of roll $(\mathrm{g}, \mathrm{h})$; estimated motion spectra and corresponding contour curves of pitch (e. f) and of roll (i. j) ; all at $F_{n}=0.275$ 
functions of heave (in $\mathrm{m} / \mathrm{m})$ ( $6 \mathrm{c}$ and $6 \mathrm{~d}$ ), vertical acceleration (in $\left.\mathrm{m} / \mathrm{sec}^{2} / \mathrm{m}\right)(6 \mathrm{~g}$ and $6 \mathrm{~h}$ ) pitch (in $\mathrm{deg} /$ $\mathrm{m})(7 \mathrm{c}$ and $7 \mathrm{~d})$ and roll (in $\mathrm{deg} / \mathrm{m})(7 \mathrm{~g}$ and $7 \mathrm{~h})$ for $\mathrm{Fn}=$ 0.275 have been shown. Here the transfer functions are not non-dimensionalized, different from the conventional approach of representation. Hence direct application of equation (14) will result in motion spectra. Example of an assumed directional wave spectrum has been shown in Fig. 6 a \& 7 a and the corresponding contour curves in Fig. 6 b \& 7 b. Using these transfer functions and the assumed wave spectrum, we obtain the motion spectra as shown in the form of heave spectrum (6e) and corresponding contour curves (6f), pitch spectrum $(7 \mathrm{e})$ and corresponding contour curves $(7 \mathrm{f})$, roll spectrum (7i) and corresponding contour curves $(7 \mathrm{j})$, acceleration spectrum (6i) and corresponding contour curves $(6 \mathrm{j})$. All the figures mentioned above have been drawn on absolute wave frequency base.

Similar caluclations for other wave spectra of different mean periods have been carried out and the significant motions have been estimated by following relationship :

$$
\begin{aligned}
& Z_{1 / 3}=4.0 \sqrt{m_{0}} \\
& \text { where } m_{0}=\int_{-\pi}^{\pi} \int_{0}^{\infty} S_{z}\left(\omega_{0}, \chi, V\right) d \omega d \chi
\end{aligned}
$$

These results for the case of $F_{n}=0.2$ and $F_{n}=0.275$ have been summarized in Fig. 8. where ratio of significant motion and significant wave height has been plotted against mean wave period $\left(T_{02}\right)$. Curves with broken line represent results in long crested wave (L. C.), curves with single dot chain represent results in narrow spreading wave (N.S.) and that with continuous line represent results in wide spreading wave (W. S.). Experimental results in long crested (marked by *), in narrow spreading (marked by $\bigcirc$ ) and in wide spreading wave (marked by $x$ ) have also been shown for comparison. The calculated results show good agreement with experimental results and the degree of deviation from conventional NSM results (only the case of wide spreading wave has been shown here) is relatively large for the cases other than heave. The order of heave increases sharply over mean wave periods of $4.0 \mathrm{sec}-15.0 \mathrm{sec}$ and stabilizes over longer wave period. This trend is similar to frequency-transfer function relation. Pitch is maximum in the region of $9.0-11.0 \mathrm{sec}$ of mean wave period. Over a wave period of $4.0 \mathrm{sec}-9.0 \mathrm{sec}$ pitch increases with directional spreading, but the relationship reverses over wave period of $9.0 \mathrm{sec}-18.0 \mathrm{sec}$. There trends may also be observed in experimental results. The higher order of heave and pitch over the wave period of $8.0-10.0 \mathrm{sec}$ in head sea is due to the fact that peak of encounter wave spectrum at this region is closer to the natural frequencies of heave and pitch. Vertical acceleration follow the same pattern as that of pitch. The effect of directional spreading is not significant in case of heave, pitch and vertical acceleration and also there is no significant variation with change in speed.

Roll gradually increases with wave period and reaches maximum at around $15.0 \mathrm{sec}$ of mean wave period which is close to natural period of roll in running condition. It can be concluded clearly from this results that the effect of directional spreading cannot be neglected in case of roll. Also the deviation from conventional NSM result is significant. This deviation is resulted from the characteristic difference in transfer functions shown in Fig. $7(\mathrm{~h})$ from the NSM results.

\section{2 Long Term Prediction}

Long term probability of motions in Winter North Atlantic has been investigated using Walden's data. Assuming Rayleigh probability density function, the probability that the amplitude of motion to exceed a value $\mathrm{M}$ is given by :

$$
q_{M}=\exp \left\{\frac{-M^{2}}{2 m_{0}}\right\}
$$

The total probability over all wave heights and wave periods for a particular heading is given by :(6)

$$
Q_{M}=\int_{0}^{\infty} \int_{0}^{\infty} \exp \left\{\frac{-M^{2}}{2 m_{0}}\right\} p(H, T) d H d T
$$

The term $p(H, T)$ in the above equation has been taken from Walden's data of wave frequency in North Atlantic Winter ${ }^{(6)}$.

Long term probability distributions of heave, pitch, roll and vertical acceleration in head sea with $F_{n}=0.2$ and $F_{n}=0.275$ have been presented in Fig. 9 where the vertical axes represent single amplitudes of motions in absolute scale. Horizontal axes represent logarithm of the probability to exceed this amplitude. The nomenclature follows the same order as that in Fig. 8. From this figures we can see that the variation of long term probability over directional spreading is not significant in case of heave, pitch and vertical acceleration; in case of roll, however, this effect cannot be neglected. Considering the natural period of heave and pitch we can read from this figure that the ship may experience in 23 years period (around $\mathrm{LOG}_{10} Q_{m}=-8.0$ ) a maximum heave amplitude of $14.5 \mathrm{~m}$ at $F_{n}=0.2$ and $18.5 \mathrm{~m}$ at $F_{n}=0.275$ and a maximum pitch amplitude of $16 \mathrm{deg}$. at $F_{n}=0.2$ and $17 \mathrm{deg}$. at $F_{n}=0.275$. The maximum roll amplitude the ship may experience in 23 years period ( $\mathrm{LOG}_{10} Q_{m}=-7.6$ ) is around $32 \mathrm{deg}$. in wide spreading and is around $24 \mathrm{deg}$. in narrow spreading wave. The variation with ship speed is not significant.

\section{Conclusion}

In this paper we have presented a practical method to estimate directional transfer functions of motions from the experimental results of motion spectra. Usually, it is said that estimation of ship motions by Strip Method (here we have used NSM) is fairly good compared to experimental results. However in our research, we have observed that the spectral definitions of motions calculated by NSM differ from experimental results. This suggests discrepancies in directional responses between 

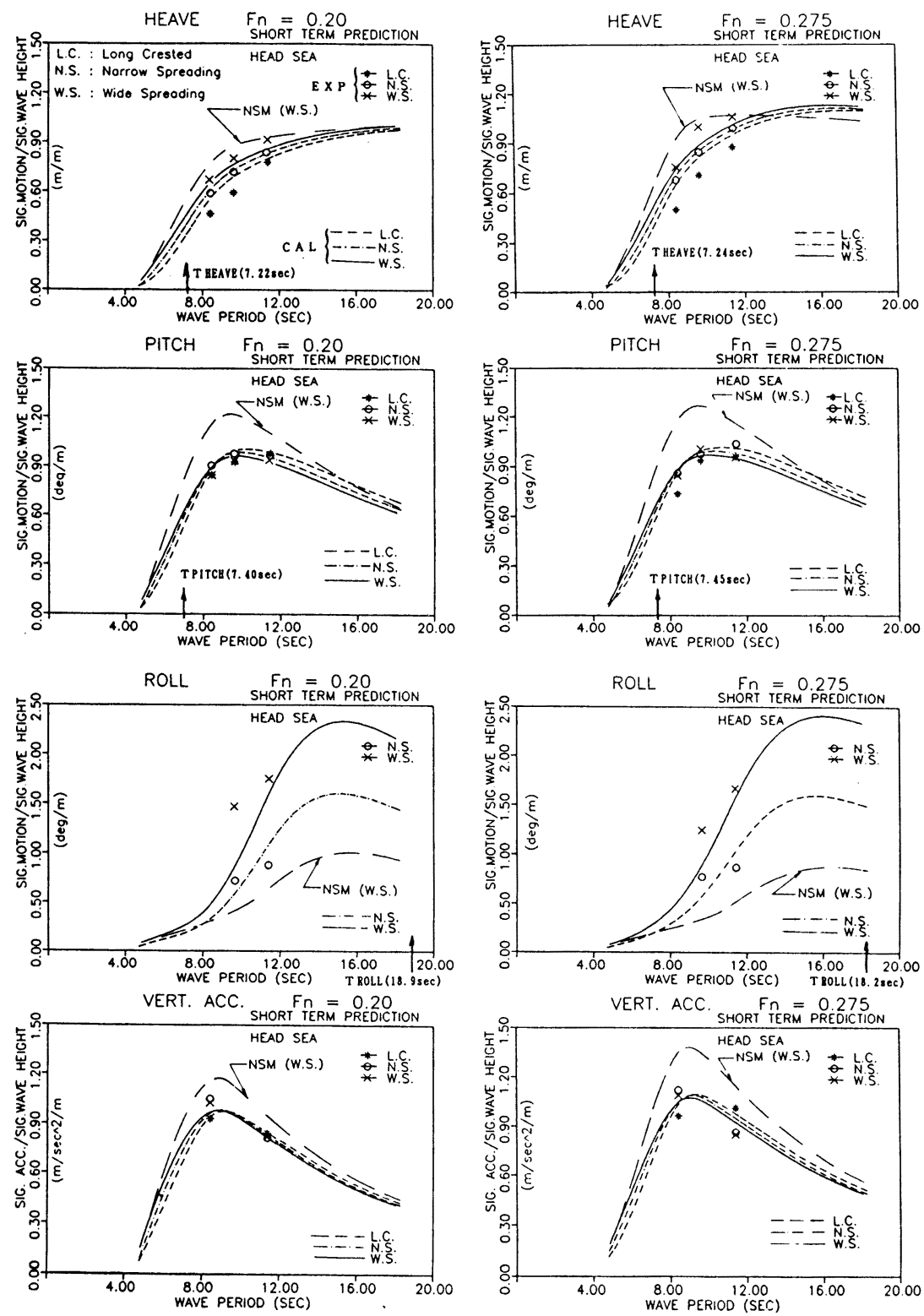

Fig. 8 Short term prediction of ship motions in head sea. Significant wave height has been taken as $6.5 \mathrm{~m}$

Strip Method and experiment. Hence the true order of transfer functions of motions must be made clear. Though the approach suggested in this paper to estimate directional transfer fnuctions of motions needs extensive model testing at the laboratory making it impractical to use at the preliminary stage of design, we hope that this will have many applications at detail design stage and will enable designer to make more rational decisions on ship form and structure. With the estimated directional transfer functions we have carried out short term and long term predictions of ship motions and the results have been presented in this paper. From various topics discussed in the present paper the conclusion may be summarized as follows:

a) The estimated transfer functions of motions obtained by the practical method suggested in this paper show good agreement with results of long crested transient water wave experiment, suggesting reliability 

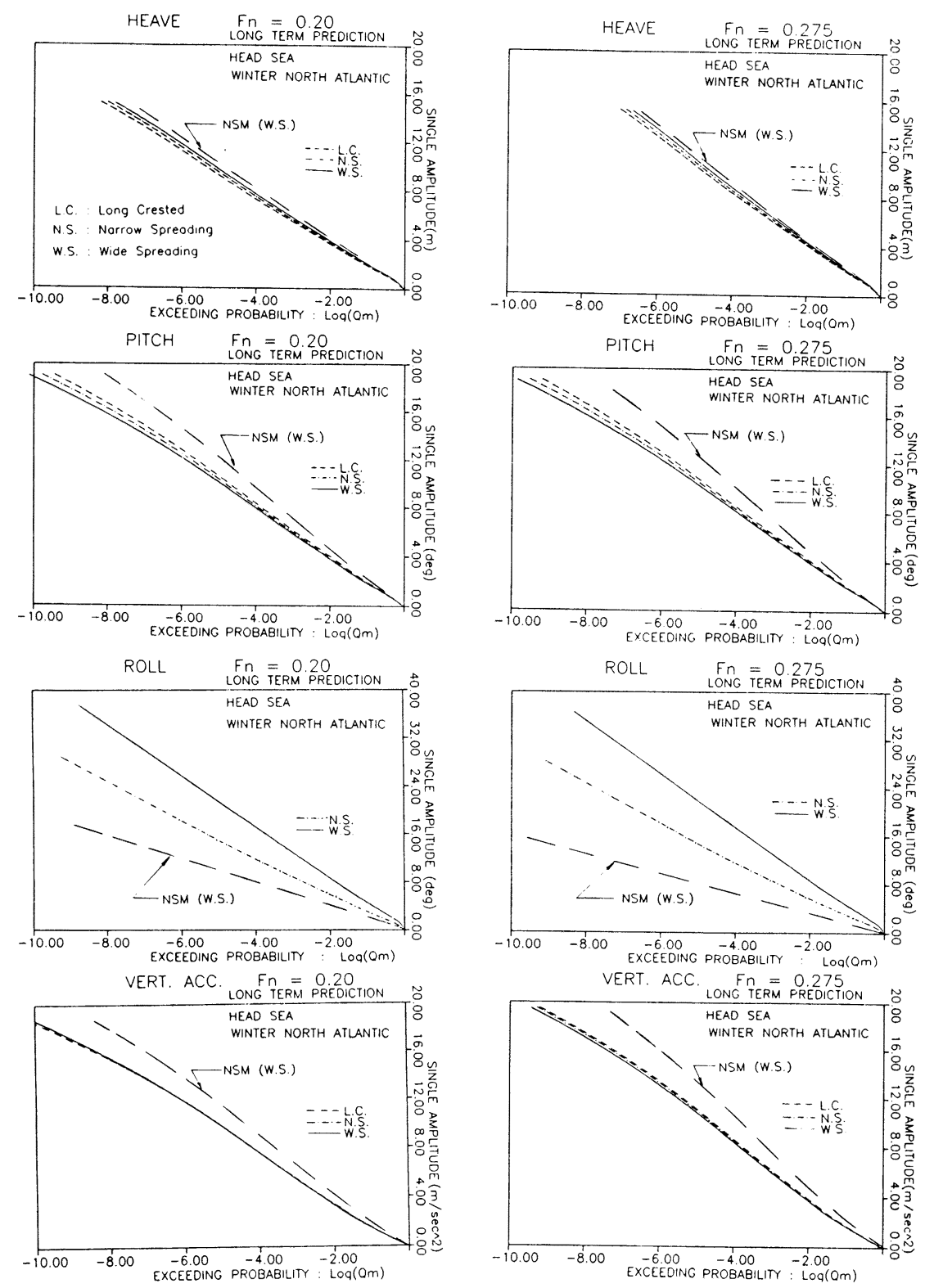

Fig. 9 Long term prediction of ship motions in Winter North Atlantic under head sea condition.

of present approach.

b) The results of directional encounter wave spectra obtained by suggested application of MLM on wave data at running condition show good agreement with the same transformed from analyzed spectra at rested condition, suggesting practicability of our approach.

c) Results of short term prediction at the points where experimental data are available are in good agreement with that of experiment. Hence the estimated transfer functions by the present approach are practically usable for statistical prediction of ship's response, at least for directional head sea condition.

d) In case of heave, pitch and vertical acceleration the effect of directional spreading on short term and long term prediction is not significant. However, this effect cannot be neglected in the case of roll.

e ) The short term prediction of roll at shorter mean wave period apparently shows under estimation if compared with experimental results. This suggests that our assumption of independency of correction factor $(\alpha)$ 
with wave encounter angle may not be truly applicable to estimate transfer functions of roll. Further investigations on this subject are thus necessary.

\section{Acknowledgement}

The authors would like to appreciate the great cooperation and invaluable suggestions received from $\mathrm{Mr}$. K. Miyakawa and Mr. T. Takayama in preparation of experimental set up and execution of experiment. The authors acknowledge the time to time help received from graduate and under graduate students of Floating Body Motion Laboratory in Yokohama National Univerity during compilation of experimental results. The authors also acknowledge the contribution of $\mathrm{Mr}$. K. Kobayashi who developed the original computer program of NSM and the program to prepare digital signal of wave maker.

\section{References}

1) Takezawa, S., Hirayama, T., 'Towed Ship Motion Test in Directional Spectrum Waves in a Long Tank', Journal of Society of Naval Architects, Japan, No. 165, 1989.
2) Takezawa, S., Hirayama, T., Acharyya, S., 'Towed Ship Motion Test in Directional Spectrum Waves in a Long Tank (Part 2)', Journal of Society of Naval Architects, Japan, No. 166, 1989.

3) Takezawa, S., Kobayashi, K., 'Directional Irregular Waves Generated in a Long Tank', Journal of Society of Naval Architects Japan, No. 163, 1988.

4) Takezawa, S., Miyakawa, K., Takayama, T., Itabashi, M., 'On The Measurement of Directional Wave Spectra by the New Wave Measuring System using Laser Beams', Journal of Society of Naval Architects, Japan, No. 166, 1989.

5) Maeda, H., Kasahara, A., 'Generation and Analy. sis of 2-Dimensional Irregular Water Waves', Journal of Kansai Society of Naval Architects, Japan, No. 202, 1986.

6) Fukuda, J., 'Statistical prediction of ship responses', Symposium on Seakeeping organized by Society of Naval Architects, Japan, July, 1969.

7) Hirayama, T., 'Real-Time Estimation of Sea Spectra Based on Motions of a Running Ship (2nd Report) - - - Directional Wave Estimation --', Journal of Kansai Society of Naval Architects, Japan, No. 204, 1987 